\title{
When scientific communities intertwine
}

\author{
Pauline Ezanno based on reviews by Arata Hidano, \\ Rowland Raymond Kao and 1 anonymous reviewer
}

\section{Open Access}

Published: 6 January 2020 Copyright: This work is licensed under the Creative Commons Attribution-NoDerivatives 4.0 International License. To view a copy of this license, visit http://creativecommons.org/licen ses/by-nd/4.0/

\section{A recommendation of:}

Delphine Destoumieux-Garzón, Pascal Bonnet, Céline Teplitsky, François Criscuolo, Pierre-Yves Henry, David Mazurais, Patrick Prunet, Gilles Salvat, Philippe Usseglio-Polatera, Etienne Verrier and Nicolas Friggens. OneARK: Strengthening the links between animal production science and animal ecology (2020) zenodo, 3632735, ver. 6 peer-reviewed and recommended by Peer Community in Animal Science. 10.5281/zenodo.3632731

Submitted: 05 July 2019, Recommended: 06 January 2020

Cite this recommendation as:

Pauline Ezanno (2020) When scientific communities intertwine. Peer Community in Animal Science, 100003. 10.24072/pci.animsci.100003

Scientific research can be seen by some as a competitive territory: competition of opinions, concepts, publications, competition for funding. Fortunately, it is above all a territory of sharing and cross-fertilization of ideas. It is gradually becoming a territory of productive interdisciplinary collaborations, despite persistent resistance to making borders more permeable [1]. At the crossroads of worlds, many challenges must be met for communities to understand each other, to be able to communicate with one another, and to benefit mutually from scientific interactions [2].

Delphine Destoumieux-Garzon and co-authors [3] propose to stimulate a single Animal Research Kinship (OneARK) to promote the crossing of the 
scientific communities in animal production and animal ecology. These two communities share many concepts and methods, which, while they are based on marked specificities (natural versus artificial systems), also and above all have common points that need to be explored more closely. Seven concepts of shared interest to improve the resilience and sustainability of animal population systems were explored by the authors: selection, system viability, system management, animal adaptability, inter-individual diversity in systems, agroecology, and animal monitoring.

This foundation stone paves the way for a finer integration between these two communities, which are close and yet distant, and which are slowly getting to know, understand, and recognize each other.

\section{References}

[1] Ledford, H. (2015). How to solve the world's biggest problems. Nature, 525, 308-311. doi: $\underline{10.1038 / 525308 \mathrm{a}}$

[2] Knapp, B., Bardenet, R., Bernabeu, M. O., Bordas, R., Bruna, M., Calderhead, B., ... Deane, C. M. (2015). Ten simple rules for a successful cross-disciplinary collaboration. PLoS Computational Biology, 11(4), e1004214. doi: 10.1371/journal.pcbi.1004214 [3] Destoumieux-Garzón, D., Bonnet, P., Teplitsky, C., Criscuolo, F., Henry, P.-Y., Mazurais, D., ... Friggens, N. (2020). OneARK: Strengthening the links between animal production science and animal ecology. Ver 6 Peer-Reviewed and Recommended by PCI Animal Science. doi: $\underline{10.5281 / \text { zenodo. } 3632731}$

\section{Revision round \#2}

2019-12-17

With the two reviewers, we have now assessed your revised preprint. As you will see, both reviewers are happy with your revisions. They have a few additional suggestions that you might be willing to consider. Once these remarks have been addressed, I will be happy to recommend your preprint.

\section{Reviewed by Rowland Raymond Kao, 2019-12-09 14:27}

My thanks to the authors for their detailed response and I think the paper is considerably improved. There are points where I think the analogies made appear sensible, but are a bit too generic and therefore could be strengthened. These comments are in the attached file - along with a few suggestions for readability and grammar.

\section{Download the review (PDF file)}

Reviewed by Arata Hidano, 2019-12-17 10:22 
Thank you for considering my comments. The revised manuscript explains the discussions points in a manner that people outside ecology can understand. This will definitely increase the impact of this paper. I would like to suggest two further points as can be found below.

Line 152 "For example, because genotypes can perform differently..." I appreciate the authors' revision to add explanations about why it is important to understand how populations respond to new artificial selection pressures. While I understand what the authors are trying to mean, the added texts are slightly confusing/redundant and I suggest a re-organisation between Line 149 and 171 . These discussions argue, I believe, the importance of understanding on the interrelationship between 'changing environment', 'selection pressure', and 'population response'. In my view, it would be much easier to follow arguments in Line 149-171 if this objective of discussion is clearly highlighted in the beginning (e.g. around Line 149 before "Understanding how populations respond to..."). Currently, different reasonings go parallelly in the text (e.g. Line 159, 161, 167), which makes it hard to understand this whole paragraph.

Line 617 "In that sense, several points discussed in this article may be..." The authors' efforts to add specific examples on how one or more of discussed points in this paper are related to avian/swine flu are appreciated. Nevertheless, while Line 553-616 are entirely specific to Influenza, Line 617 and onwards are very general. This mismatch makes it less convincing why the marriage between animal production and ecology is important. Perhaps the way to overcome is to make discussions from Line 617 more specific to Influenza. I agree with the idea that the use of new technologies for monitoring animal contacts, adapting livestock managements, and increasing genetic diversities are helpful in tackling avian/swine flu. Immune priming is also an interesting topic. Therefore, it would be very nice to explain how each of these topics could potentially facilitate controlling influenza. For instance, how does animal diversity help system resilience in terms of minimising the impact of Influenza? Maybe you could also deepen discussions around employing new technologies, say, for wild bird migrations? Or what kind of livestock management do we need to minismise the impact of Influenza?

Line 136: Typo 'resilience'

\section{Author's reply:}

our responses are in the attached files (I regrouped responses to both reviewers in the 1 file)

Download author's reply (PDF file)

\section{Revision round \#1}

2019-08-01

With two reviewers, we have now assessed your manuscript. As you will see, both reviewers found your manuscript very interesting, cross-disciplinary integration being crucial and the interface between livestock production and animal ecology really needing a particular focus. However, they also have made insightful remarks which should help you enhance the clarity of your manuscript and improve its significance. Please address these points and submit a revised 
version of your manuscript together with a response letter. I will be happy to assess this revised manuscript for recommandation.

Reviewed by Rowland Raymond Kao, 2019-07-31 20:26

Download the review (PDF file)

Reviewed by anonymous reviewer, 2019-07-22 03:06

Download the review (PDF file)

Author's reply:

Dear Editor,

Below we have provided full responses (in red text) to each of the two reviewers in the attached pdf file, and would like to thank them for their useful comments which we very much appreciated. In order to accommodate these comments we have added some text as well as a number of references which should help the interested reader to be able to follow up on some of the issues raised. We sincerely hope that this revision meets your requirements and look forward to your, hopefully positive, response.

Sincerely Nicolas Friggens, Delphine Destoumieux, Pascal Bonnet

Download author's reply (PDF file) 